\title{
Robotic Approach to Colon Resection
}

Joshua A. Waters MD

Assistant Professor

Department of Surgery

Indiana University School of Medicine

1801 N. Senate Blvd

Indianapolis, IN 46202

jwaters1@iuhealth.org

Todd D. Francone, MD MPH FACS

Department of Colon and Rectal Surgery

Lahey Hospital \& Medical Center

Assistant Professor of Surgery

Tufts University Medical Center

41 Mall Road

Burlington, MA 01805

todd.d.francone@1ahey.org

\begin{abstract}
Robotic surgical techniques are being increasingly adopted as a tool in the minimally invasive armamentarium of the colorectal surgeon. These platforms present numerous potential advantages in visualization, precise dissection, and tissue manipulation while potentially reducing operator fatigue. They may also reduce the learning curve and rate of conversion, though the short and long-term benefits of this approach in non-pelvic colorectal surgery, and the cost-benefit balance remain an ongoing debate. Adherence to established principles of laparoscopic colon surgery, a robust understanding of the operative anatomy, and proper patient preparation and setup are critical for the efficient and effective utilization of a robotic approach for colon resection.
\end{abstract}

Keywords - colon, colectomy, minimally invasive, robotic surgery, laparoscopy,

This is the author's manuscript of the article published in final edited form as:

Waters, J. A., \& Francone, T. D. (2016). Robotic approach to colon resection. Seminars in Colon and Rectal Surgery, 27(3), 136-143. https://doi.org/10.1053/j.scrs.2016.04.005 


\section{Introduction}

Minimally invasive approaches to colon resection have revolutionized the practice of colon and rectal surgery. Although the safety, efficacy, and efficiency of these approaches have been repeatedly validated in the literature, the prevalence of laparoscopy in colon resection has reached a relative plateau. ${ }^{1}$ Robotic surgical platforms provide an alternative minimally invasive approach that addresses the current limitations of straight laparoscopic colon resections. For other surgical disciplines, particularly urology, robotic approaches have become an accepted, if not integral part of their operative armamentarium. This is in part related to the potential advantages inherent to robotic surgery. These include improvements in overall visualization due to improved depth assessment as well as a stable operator controlled field of view. Surgeon control of three independent instruments with improved articulation and degrees of freedom is an additional advantage. Furthermore, a fixed abdominal wall fulcrum purportedly reduces abdominal wall trauma. Despite these advantages there is a relative paucity of literature supporting the positive impacts on patient outcome of robotic colon resection compared to conventional laparoscopy, and the technology remains saddled with several notable limitations including cost, setup time, and a new technical learning curve. ${ }^{2}$

In this review, we will provide an update on the current state of literature regarding robotic colon resection, provide tips on how to effectively prepare and position the patient, and plan port placement. Lastly, we will address the operative approach and pitfalls encountered for right and sigmoid colectomy. 


\section{Indications}

In the absence of the cost-effective conundrum associated with robotic surgery, the spectrum of indications for a robotic colon resection essentially mirror that of conventional laparoscopy. The breadth of colorectal pathology can be reasonably approached robotically, including both benign inflammatory disease such as diverticulitis and inflammatory bowel disease. ${ }^{3}$ Similarly, roboticassisted colectomy for neoplasia has been widely employed stemming from the acceptance of laparoscopy utilization for oncologic resections. ${ }^{4,5}$ Current literature suggests at least equivocal short-term oncologic outcomes such as minimal tumor manipulation, high vascular ligation, and mesenteric lymphadenectomy when performing right and left colectomy. ${ }^{2}$ The question remains whether the technical advantages of robotic surgery will translate not only into improved longterm oncologic outcomes but press forward the adaption of minimally invasive techniques for oncologic colon resections.

Relative contra-indications to robotic surgery are similar to that for laparoscopy including patients with multiple prior open abdominal operations and prohibitive adhesive disease, profound inflammatory processes, locally advanced malignancy, physiologic inability to tolerate insufflation pneumoperitoneum or extreme positioning. As always, the choice of operative technique (MIS vs. open) as well as the tool (robotic, laparoscopic, hand-assist surgery) is left to the discretion of the operative surgeon. A fundamental understanding of the surgical anatomy and various approaches is essential and allow the surgeon the ability to proceed in a safe manner, perform an appropriate oncological resection when necessary, and allow for additional diagnostic and therapeutic maneuvering. 


\section{Current evidence and outcomes}

There is a plethora of data suggesting a potential benefit to robotic surgery in the setting of colon and rectal surgery including shorter length of stay, less pain, lower conversion rates and equivalent oncologic outcomes. ${ }^{2}$ However, the majority of current data for both segmental colectomy and proctectomy are small case series and retrospective in nature limiting their ability to draw concrete conclusions. That being said, the theoretical advantages of utilizing roboticassisted surgery within the confined space of the bony pelvis, such as with a low anterior resection, are widely accepted. This may in part be related to the reality that minimally invasive surgery (MIS) in the pelvis is particularly challenging no matter the selected technique (laparoscopic, hand-assist or robotic). As a result, there remain concerns regarding the ability to achieve equivalent oncologic outcomes in the deep pelvis when performing a minimally invasive proctectomy and as a result, the adaptation for MIS for rectal cancer has been underwhelming. ${ }^{6,7}$

On the contrary, laparoscopic segmental colectomy, right or left, is considered to be somewhat less challenging than a pelvic dissection. As a result, it is considered more easily reproducible with consistent results and at a lower cost. Subsequently, the advantages of robotic surgery for a segmental colectomy are justly questioned. A critical review of the evidence addressing the role of robotic segmental colectomy is particularly relevant given the substantial up-front and recurring cost associated with a robotic surgical system.

The role of robotic colorectal surgery has been examined over the past 2 decades and has been shown to be a feasible and safe procedure. However, increased operative time and the overall additional expense of robotics were an early concern. ${ }^{8}$ Recent case series have found similar results (Table 1). Park et. al. conducted a randomized trial comparing 70 patients 
undergoing a robotic or laparoscopic right colectomy for malignancy. They found no difference in oncologic parameters, pain, or early postoperative complications. Of note, they found the robotic approach was associated with longer operative time (approximately one hour) and higher overall hospital cost (by approximately $20 \%$ ). ${ }^{9}$

Casillas et. al report an impressive single-surgeon experience comparing conventional laparoscopic and robotic approaches to left or right colectomy. This non-randomized comparison of over 300 colectomies revealed an average increase in the operative time of one hour for both left and right colectomy. Furthermore, they also reported a reduced length of stay ( 3.6 vs 6.5 days) for robotic left colectomy but not right colectomy. Interestingly, they also noted a lower rate of postoperative ileus in the robotic group. ${ }^{10}$

A recent study utilizing the American College of Surgeons NSQIP database compared outcomes for laparoscopic and robotic colorectal procedures in 2013. As expected, the study reported lower conversion rates for pelvic robotic procedures (10\% vs $13 \%)$; however, this was not demonstrated for non-pelvic colectomies (approximately 4\% in each group). Although there was no difference in complication rates, increased operative time (167 vs. 211 minutes) and a one day reduction in length of stay (5 vs 4 days) was reported for robotic colectomy. ${ }^{11}$.

In summary, the use of robotics for segmental colectomy is unsubstantiated due to the limitations of not only the current literature but also the current technology. The current literature is represents robotic assisted colectomy with dated technology utilizing the S or Si Da Vinci robotic systems. These models are quite limited when performing multi-quadrant surgery especially when mobilization of the flexure is required. The newer Xi platform, has the demonstrated a tangible potential to enable multi-quadrant surgery, especially with facilitating 
the docking process, minimizing the need for re-docking and repositioning due to limited or difficult exposure at the hepatic or splenic flexure.

\section{Patient preparation}

Preoperative preparation of the patient for robotic colon resection should follow along similar lines as conventional laparoscopic colectomy. We suggest routine mechanical and oral antibiotic bowel preparation for left-sided resections, and selective use of bowel preparations for right colectomy based on institutional/group practice. The exception for this would be in the setting of planned intracorporeal anastomosis for right colectomy. In these cases, purgative bowel preparation should be utilized to reduce peritoneal contamination and improve handling.

As with all robotic surgery, efficiency relies heavily on patient setup and positioning. The skilled laparoscopist utilizes a dynamic surgical bed to vary the patient's position throughout different phases of conventional laparoscopic colectomy, whereas the currently available iterations of robotic surgical systems do not allow repositioning of the operating table without un-docking of the robot. For this reason, multi-quadrant surgery is more challenging with current robotic surgery such that proper positioning and port-set up are critical to operative success.

Modified low-lithotomy or split-leg positioning can be utilized for all colon resections. The patient should be secured on a padded "bean-bag" or other non-slip device, with arms tucked at the patients side. We suggest further securing the patient with 3-inch silk tape at the level of the nipple wrapped circumferentially around the bed allowing for positioning without unwanted patient movement (Figure 1). 


\section{Port placement and docking}

After securing the patient and prior to prepping or draping, it is our practice to map and mark the relevant abdominal features and anticipated port positioning. The anterior superior iliac spine, costal margins and potential extraction sites or stoma marks should be identified. Notably if the camera is stationed too close to the relevant anatomy, collisions will be encountered. Additionally, the anticipated port locations must be reconsidered after insufflation as the abdominal topography will change variably following pneumoperitoneum. An initial laparoscopic overview is performed in every procedure. The larger robotic camera is quite cumbersome and heavy, not to mention costly to repair if dropped, so we discourage its use for this portion of the case. In contrast, the opening of a $5 \mathrm{~mm}$ laparoscope and other laparoscopic equipment may add unnecessary cost to the procedure. By avoiding the use of unnecessary equipment, expenses are lowered. We prefer to utilize the $5 \mathrm{~mm}$ laparoscopic camera as it is easier to maneuver, especially when visualizing ports in the upper quadrants. This allows for rapid and effective visualization of the abdomen for altered anatomy and efficient preparation of the operative field prior to docking including direct visualization of the robotic instruments. Once all ports are placed, the camera visualizes careful insertion of robotic instruments, all pointed towards the pelvis.

Of note, the port placement and docking strategies outlined are those typically utilized for DaVinci S or Si platforms. The newer Xi system employs a boom mounted arm design which allows a more straightforward linear port placement strategy, and facilitates docking from any position around the patient. 


\section{Right colectomy}

For robotic right colectomy a four arm technique is typically used (Figure 2) although a three arm technique is also feasible (Figure 3). A $12 \mathrm{~mm}$ trocar is placed in the midline above or below the umbilicus depending on the length of the patient's torso. Entry to the peritoneal cavity is gained via the surgeon's preference including an open cut-down technique in the supra-umbilical position, Veress needle or optical entry techniques. Three additional $8 \mathrm{~mm}$ robotic ports are placed in the subxiphoid (arm \#3), left upper quadrant (arm \#2) and suprapubic (arm \#1) positions. A $5 \mathrm{~mm}$ assist port is placed in the left lower quadrant (Figure 2).

The operating table should then be positioned in moderate Trendelenberg and left lateral decubitus rotation. The omentum should be elevated cephlad to the transverse colon. The small bowel should be swept into the left hemi-abdomen. Once the target anatomy is visualized and the ileocolic pedicle is confirmed, the docking of the robot may commence. The robot is positioned directly over the patient right, with a slight cranial bias.

\section{Sigmoid colectomy}

As with a right colectomy, mobilization of the descending and sigmoid colon requires fastidious attention to positioning and port placement. Extra care directed at these steps will pay dividends in maintaining an efficient robotic dissection given the need for multi-quadrant dissection. Figure 4 demonstrates a single-dock robotic left colectomy. The $12 \mathrm{~mm}$ camera port is placed 1$2 \mathrm{~cm}$ superior and 1-2 $\mathrm{cm}$ to the right of the umbilicus. Three additional $8 \mathrm{~mm}$ robotic ports are placed along a triangle drawn from umbilicus to either anterior superior iliac spine. Arm \#1 is placed in the right lower quadrant, while arm \#2 is placed in the left lower quadrant near the mid-clavicular line and arm \#3 in the right sub-xiphoid position. Take notice that arm 3 has 
access not only to the LUQ but also the LLQ and pelvis. It is essential that the camera is port is placed off the midline such that the arm 3 is not positioned directly behind it otherwise there will be numerous collisions while operating in the pelvis. This port system may also be used when performing a single-dock low anterior resection. If collisions do occur perhaps in a particularly deep and narrow pelvis, arm \# 3 may be re-docked to the left lower quadrant port while arm 2 is shifted medial to the mid-clavicular line in an additional port.

The patient should then be positioned in moderate Trendelenberg and right side down, facilitating the small bowel to be swept out of the pelvis and into the right abdomen. Excessive Trendelenberg positioning at this point, may result in settling of the small bowel in the left upper abdomen leading to obscured visualization of the inferior mesenteric vein and splenic flexure. A this point, the robot may be docked at approximately a 45 degree angle over the left hip. Close attention should be paid at this time to arm spacing and positioning.

\section{Technical considerations}

\section{Right colectomy}

The most common approach utilized for a minimally invasive right colectomy is medial to lateral dissection. This dissection relies on the effective entry into the embryologic fusion plane between the right colonic mesentery and the retroperitoneum. Orientation to key structures in this dissection can be undertaken by grasping the cecum or its mesentery with arm \#3 and placing antero-inferior traction. This will place the vascular pedicle on tension and facilitate identification of the ileo-colic vessels (Figure 6). Arms \#1 and \#2 can then be used for the majority of the medial dissection. The peritoneum investing the mesentery should be scored parallel to the ileo-colic pedicle just posterior to its fold. The correct avascular plane should lie 
immediately posterior to the vessel. Importantly, the duodenum will come into view almost immediately to the right at the base of the pedicle. A combination of blunt posterior sweeps and sharp dissection can be undertaken to develop this space working laterally (Figure 7). The lacy vessels of the retroperitnoneum should be swept down, taking care to identify and tread carefully near the duodenum, particularly given the lack of haptic feedback with the robot. Visual cues should be utilized to assess tension and tissue effect. As the dissection proceeds laterally, a clear window of peritoneum is encountered just beyond the ileo-colic pedicle. At this point, the duodenum should again be noted and swept posteriorly. When this is adequately clear, the pedicle can be ligated. Of note, the pedicle often acts as a point of tension which can facilitate the medial to lateral dissection by allowing counter-tension for posterior sweeping of the retroperitoneum. For this reason, we perform the majority of the medial to lateral dissection with pedicle intact. Importantly though, the lack of haptic feedback can make undue tension on the undivided pedicle a possibility, and should be monitored closely. For division, the robotic vessel sealing device or an additional laparoscopic energy device placed through the assistant port (Figure 8 ). We suggest having an alternative method readily available for quick control of the pedicle in the event of failed sealing such as robotic clips or Endoloop ${ }^{\mathrm{TM}}$ device. The medial to lateral dissection is then further developed cephalad toward the hepatic flexure and medially toward the duodenum.

Next, the right colon is mobilized off the right pelvic sidewall. The cecum is retracted medially utilizing arm \#3. The avascular cecal and terminal ileal attachments to the right pelvic inlet are visualized. The prior medial dissection is often immediately encountered by visualization of a purplish discoloration in the attachments between the side wall and the cecum. Division of the attachments gains entry into the previous medial to lateral plane. Monopolar scissors in arm \#1 
and counter-traction with arm \#2 will facilitate dissection up toward the lateral attachments. Here, the dissection is continued toward the hepatic flexure using progressive anteromedial traction. At this point, we find that approaching the hepatic flexure from the transverse colon is often more effective.

The omentum can be grasped and elevated toward the abdominal wall using arm \#3, while arm $\# 1$ and \#2 gain entry to the lesser sac, as identified by the posterior aspect of the stomach (Figure 9). The dissection should be initiated at the location at which the falciform ligament crosses the transverse colon. As the dissection is carried toward the hepatic flexure, the leaves of the omentum can be difficult to discern. Careful dissection should yield entry into the prior dissection plane overlying the duodenal sweep at approximately the level of the gallbladder. The remaining hepato-colic attachments should be divided. If warranted the right branch of the middle colic maybe divided intra-corporeally. This may facilitate extraction of the right colon especially for those patients with a short or thick transverse colon mesentery such as in the obese (Figure 10).

Once complete, the right colon should be mobile to its embryologic midline position. This will facilitate extraction through a midline or periumbilical incision for extracorporeal anastomosis, or tension free ileo-transverse anastomosis for intracorporeal anastomosis.

\section{Sigmoid colectomy}

Similar to the right colon, we typically address the left or sigmoid colon with a medial to lateral approach. The sigmoid colon mesentery should be placed on tension anteriorly and toward the left hip using arm \#2 to identify the inferior mesenteric artery (IMA) pedicle. The operator should also orient to the sacral promontory at this time (Figure 11). It is also important to 
determine whether the sigmoid colon is coiled, or adherent within the pelvis, as this can lead to challenges in appropriate identification of the vascular pedicle. The peritoneum can be scored using monopolar scissor inferior and posterior to the arc of the superior hemorrhoidal artery. At this point "pneumo-dissection" of the insufflated carbon dioxide will fill the avascular plane often revealing the key structures (Figure 12). Often the pelvic sympathetic nerves can be identified and swept posteriorly at this time. These serve as a key landmark of the proper plane (Figure 13). Arms \#1 and \#3 carry the burden of the dissection working in a lateral fashion immediately under the superior hemorrhoidal artery working up toward the origin of the IMA. During this dissection, the left ureter and gonadal vessels should be identified and left in the retroperitoneum (Figure 14). The presence of a localizing ureteral stent may facilitate the rapid identification of the left ureter during this step.

Following the identification of the origin of the IMA, an additional window can be developed cranially between the IMA and inferior mesenteric vein (IMV) (Figure 15). At this point, the takeoff of the left colic artery can often be identified and often gives a " $T$ " appearance of the mesentery (Figure 16). Once the vascular anatomy has been confirmed, the left colic may be preserved by ligation just distal on the superior hemorrhoidal, or sacrificed with ligation of the IMA at its origin. In either event, the ureter should once again be identified, and a robotic vessel sealer or laparoscopic energy device can be utilize to control the vascular pedicle. The avascular plane is further developed laterally and up toward the inferior border of the pancreas. It is often reasonable to divide the IMV early in this step to prevent undue tension and possible avulsion as well as avoiding the loss of timely exposure (Figure 17).

The focus of the procedure is then shifted to the mobilization of the lateral attachments of the sigmoid and descending colon (Figure 18). A robust medial dissection should facilitate this step. 
Arm \#3 and the assistant typically create medial retraction, while monopolar cautery in arm \#1 divides the lateral attachments. This dissection can be taken as cranial as possible. It is at this juncture that the limitations of earlier generation DaVinci S or Si platforms become evident particularly in patients with narrow abdominal domain or a high splenic flexure. As mentioned previously, the splenic flexure may be approached with the 3 arm in the right subxiphoid port position (Figure 4). In order to avoid collisions, arm 2 may be undocked from the port and placed to the side. As with a right colectomy, mobilization of the flexure is facilitated by gaining entry into the lesser sac at the transverse colon, and working toward the splenic flexure, thereby meeting the prior lateral mobilization (Figure 9).

Upon adequate mobilization of the descending and sigmoid colon, there are numerous approaches to colonic division and creation of an stapled end-to-end anastomosis. A Pfannenstiel incision can be created 1-2 fingerbreadths above the pubic symphysis allowing for division of the upper rectum through the incision, and exteriorization of the specimen with subsequent performance of the end-to end stapling technique. Alternatively, a robotic or laparoscopic stapler can be utilized to divide the specimen intra-corporeally. This approach may facilitate a slightly smaller extraction incision or utilization of natural orifices such as the vagina or anus.

\section{Summary}

Robotic surgical platforms provide an alternative approach to conventional laparoscopic techniques for the colorectal surgeon. Although a number of theoretical advantages for nonpelvic robotic assisted colon resection have been suggested, the existing literature would at best 
support equivalency with regard to patient outcomes. Several unanswered questions remain about the overall impact from a cost and efficiency standpoint.

Despite these controversies, the techniques and principles employed to address pathology of the right or left colon remain similar to those developed for conventional laparoscopy. Medial to lateral mobilization, high vascular ligation, and adherence to embryologic tissue planes remain critical for successful conduct of the operation. Furthermore, patient positioning and port placement remain key elements to achieving a successful operative outcome. 


\section{References}

1. Moghadamyeghaneh Z, Carmichael JC, Mills S, Pigazzi A, Nguyen NT, Stamos MJ. Variations in Laparoscopic Colectomy Utilization in the United States. Dis Colon Rectum. 2015;58(10):950956.

2. $\mathrm{Xu} \mathrm{H,} \mathrm{Li} \mathrm{J,} \mathrm{Sun} \mathrm{Y,} \mathrm{et} \mathrm{al.} \mathrm{Robotic} \mathrm{versus} \mathrm{laparoscopic} \mathrm{right} \mathrm{colectomy:} \mathrm{a} \mathrm{meta-analysis.} \mathrm{World} \mathrm{J} \mathrm{Surg}$ Oncol. 2014;12:274.

3. Ragupathi M, Ramos-Valadez DI, Patel CB, Haas EM. Robotic-assisted laparoscopic surgery for recurrent diverticulitis: experience in consecutive cases and a review of the literature. Surg Endosc. 2011;25(1):199-206.

4. Group COoSTS. A comparison of laparoscopically assisted and open colectomy for colon cancer. N Engl J Med. 2004;350(20):2050-2059.

5. Jayne DG, Guillou PJ, Thorpe H, et al. Randomized trial of laparoscopic-assisted resection of colorectal carcinoma: 3-year results of the UK MRC CLASICC Trial Group. J Clin Oncol. 2007;25(21):3061-3068.

6. Stevenson AR, Solomon MJ, Lumley JW, et al. Effect of Laparoscopic-Assisted Resection vs Open Resection on Pathological Outcomes in Rectal Cancer: The ALaCaRT Randomized Clinical Trial. JAMA. 2015;314(13):1356-1363.

7. Fleshman J, Branda M, Sargent DJ, et al. Effect of Laparoscopic-Assisted Resection vs Open Resection of Stage II or III Rectal Cancer on Pathologic Outcomes: The ACOSOG Z6051 Randomized Clinical Trial. JAMA. 2015;314(13):1346-1355.

8. Delaney CP, Lynch AC, Senagore AJ, Fazio VW. Comparison of robotically performed and traditional laparoscopic colorectal surgery. Dis Colon Rectum. 2003;46(12):1633-1639.

9. Park JS, Choi GS, Park SY, Kim HJ, Ryuk JP. Randomized clinical trial of robot-assisted versus standard laparoscopic right colectomy. Br J Surg. 2012;99(9):1219-1226.

10. Casillas MA, Leichtle SW, Wahl WL, et al. Improved perioperative and short-term outcomes of robotic versus conventional laparoscopic colorectal operations. Am J Surg. 2014;208(1):33-40.

11. Bhama AR, Obias V, Welch KB, Vandewarker JF, Cleary RK. A comparison of laparoscopic and robotic colorectal surgery outcomes using the American College of Surgeons National Surgical Quality Improvement Program (ACS NSQIP) database. Surg Endosc. 2015.

12. Patel SV, Van Koughnett JA, Howe B, Wexner SD. Spin Is Common in Studies Assessing Robotic Colorectal Surgery: An Assessment of Reporting and Interpretation of Study Results. Dis Colon Rectum. 2015;58(9):878-884.

13. DeNoto $G$, Rubach E, Ravikumar TS. A standardized technique for robotically performed sigmoid colectomy. J Laparoendosc Adv Surg Tech A. 2006;16(6):551-556.

14. Rawlings AL, Woodland JH, Vegunta RK, Crawford DL. Robotic versus laparoscopic colectomy. Surg Endosc. 2007;21(10):1701-1708.

15. D'Annibale A, Pernazza G, Morpurgo E, et al. Robotic right colon resection: evaluation of first 50 consecutive cases for malignant disease. Ann Surg Oncol. 2010;17(11):2856-2862.

16. deSouza AL, Prasad LM, Park JJ, Marecik SJ, Blumetti J, Abcarian H. Robotic assistance in right hemicolectomy: is there a role? Dis Colon Rectum. 2010;53(7):1000-1006. 


\section{Figure Legends}

Figure 1: Patient set-up. Patient is placed in the split-leg position for most cases. Cases requiring a synchronous perineal approach such as an intersphincteric dissection., the patient is placed in the modified lithotomy position.

Figure 2: Four arm technique for robotic right colectomy. Extraction is site is through the midline or surgeon preference.

Figure 3: Three arm technique for robotic right colectomy. Extraction is site is through the midline or surgeon preference.

Figure 4: Single-dock port placement for a robotic sigmoidectomy or LAR. Notice the camera port is slightly off midline. This allows dissection of the splenic flexure and pelvis utilizing the $3 \mathrm{arm}$ (which is also moved slightly more medial)

Figure 5: Alternative port placement requiring re-docking of arm 3 to access the pelvis. When approaching the splenic flexure the 3 arm is located on the left side of the abdomen as in Figure 4. When the pelvic dissection is initiated, arm 3 may be re-docked to the left lower quadrant while arm 2 is shifted to the the mid-abdomen.

Figure 6: Grasping the cecum or its mesentery and lifting anterolateral to the abdominal wall will create a tenting or "bowstring" effect with a distinct crease coursing parallel to the vessel.

Figure 7: Medial dissection is initiated by sweeping the retroperitoneum down and away; immediate identification of the duodenum is critical in order to avoid injury.

Figure 8: When dividing the ileocolic artery, the surgeon must always be mindful of the duodenum.

Figure 9: Accessing the lesser sac through the omentum (gastro-colic ligament); early entry into the lesser sac is key to facilitating mobilization of the transverse colon.

Figure 10: The right branch of the middle colic vessels is often divided during a right colectomy.

Figure 11: The outline of the Inferior mesenteric artery (IMA) can be visualized by gentle ventral retraction of the pedicle as it courses of over the sacral promontory (SP). The space between the IMA and the sacral promontory marks the avascular plane between the retroperitoneum and the colon mesentery.

Figure 12: Scoring parallel to the IMA allows for entry of $\mathrm{CO}^{2}$ into the avascular areolar plane indicating the appropriate plane.

Figure 13: Pelvic anatomy highlighting the ureter, goandal vessels, sacral promontory, hypogastric nerves and avascular alveolar space between fascia propria and presacral fascia 
Figure 14: Care is taken to identify and avoid the left ureter, left gonadal vessels, and the hypogastric nerve plexus

Figure 15: Continued medial to lateral mobilization between the IMA and IMV will facilitate mobilization of the splenic flexure.

Figure 16: Mobilization cephalad and caudal to the takeoff of the IMA will give a "T" appearance; this allows proper identification of the left colic artery such that it may be preserved when necessary.

Figure 17: The IMV is identified at the inferior tail of the pancreas near the splenic flexure.

Figure 18: When dividing the lateral attachments, dissection is carried from the pelvis toward the splenic flexure; often, Arm2 becomes limited in its use due to increased collisions. 


\begin{tabular}{|c|c|c|c|c|c|c|c|c|}
\hline Study & Year & $\mathrm{N}$ & $\begin{array}{c}\text { Major } \\
\text { Morbidity (\%) }\end{array}$ & $\begin{array}{c}\text { Conversion } \\
(\%)\end{array}$ & $\begin{array}{c}\text { LOS } \\
\text { (days) }\end{array}$ & $\begin{array}{l}\text { OR Time } \\
\text { (min) }\end{array}$ & $\begin{array}{l}\text { EBL } \\
\text { (ml) }\end{array}$ & $\begin{array}{c}\text { LN } \\
\text { Harvest }\end{array}$ \\
\hline \multicolumn{9}{|c|}{ Robotic Right colectomy } \\
\hline DeNoto et al. ${ }^{13}$ & 2006 & 47 & 13 & 4 & 4.3 & 134 & 76 & NR \\
\hline Rawlings et al. ${ }^{14}$ & 2007 & 17 & NR & 0 & 5.2 & 177 & NR & NR \\
\hline D'Annibale et al. $^{15}$ & 2010 & 50 & 2 & 0 & 7 & 224 & 20 & 19 \\
\hline deSouza et al. ${ }^{16}$ & 2010 & 40 & 5 & 3 & 5 & 159 & 50 & 17 \\
\hline Casillas et al. ${ }^{10}$ & 2014 & 52 & 0 & 4 & 3.6 & 143 & 63 & 28 \\
\hline \multicolumn{9}{|l|}{ Left colectomy } \\
\hline Rawlings et al. ${ }^{14}$ & 2007 & 13 & NR & 15 & 6 & 103 & NR & NR \\
\hline Ragupathi et al. ${ }^{3}$ & 2011 & 24 & 0 & 0 & 3.4 & 210 & 90 & NR \\
\hline Casillas et al. ${ }^{10}$ & 2014 & 68 & 0 & 4 & 6.2 & 188 & 89 & 20 \\
\hline
\end{tabular}

NR - not reported; LN - lymph node; EBL - estimated blood loss; LOS - length of stay 
Figures

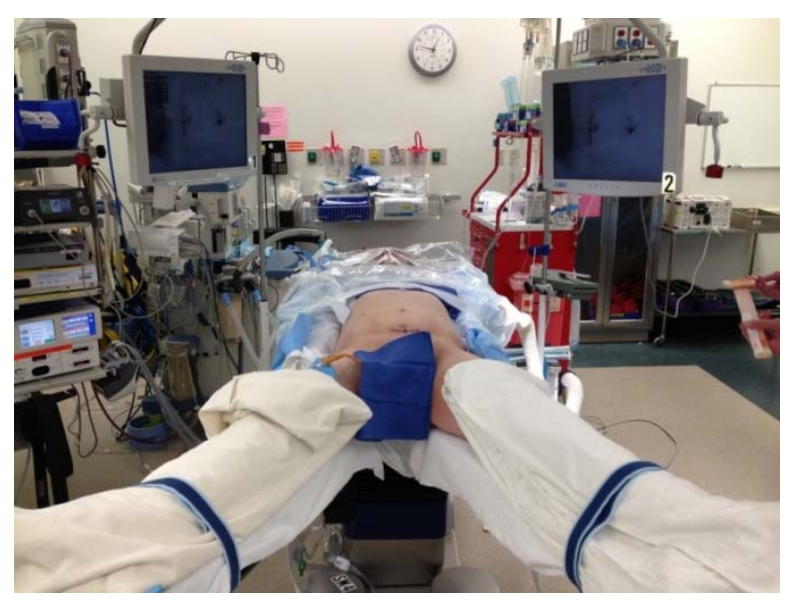

Figure 1

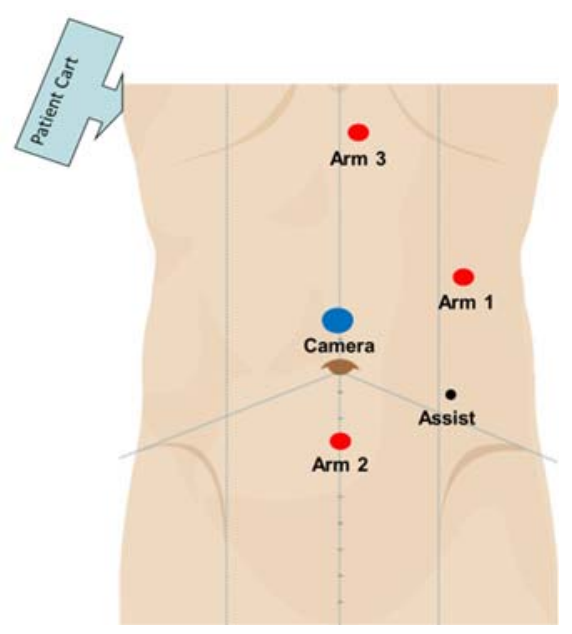

Figure 2

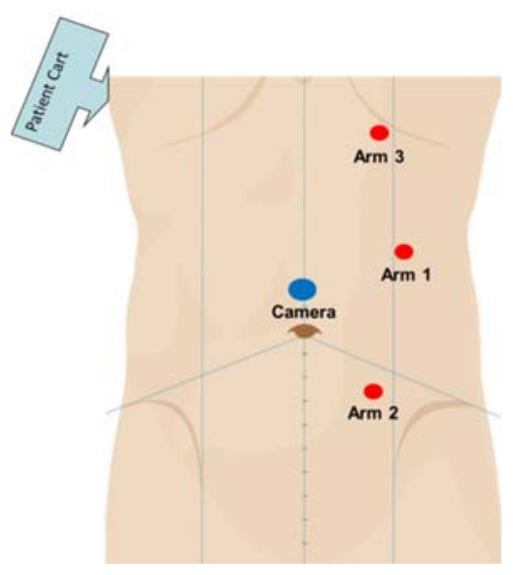

Figure 3 


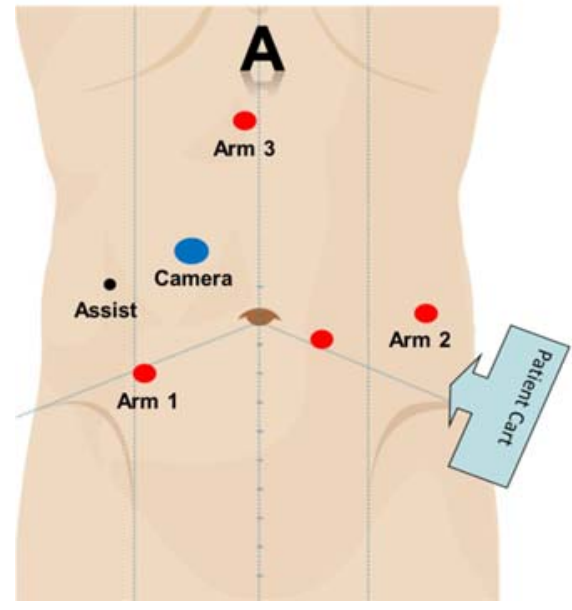

Figure 4

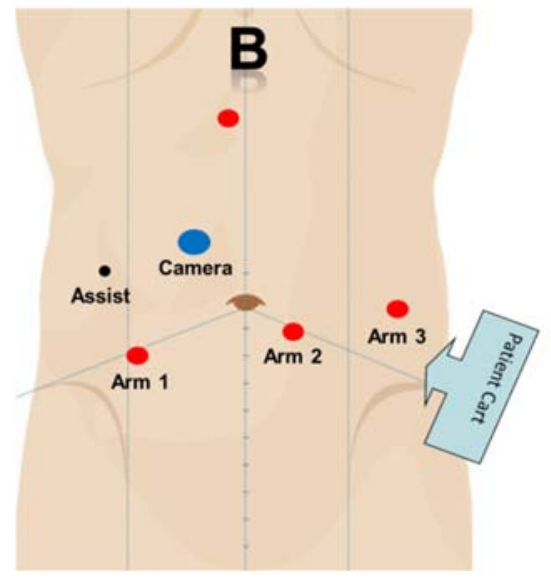

Figure 5

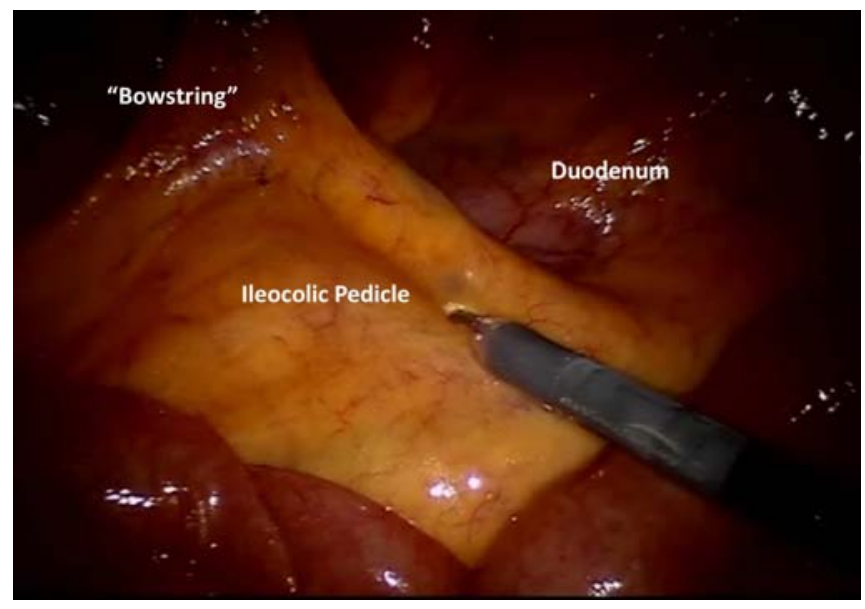

Figure 6 


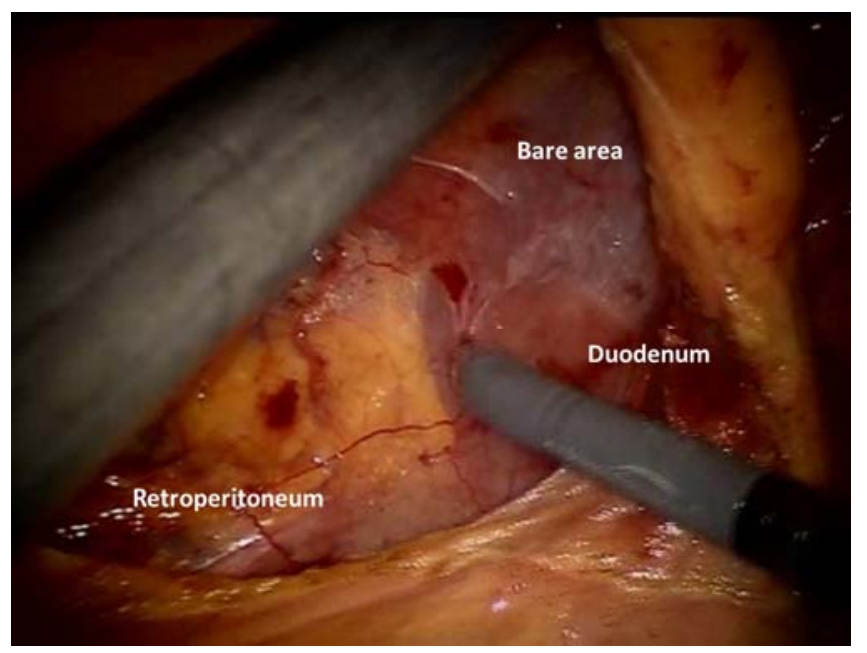

Figure 7

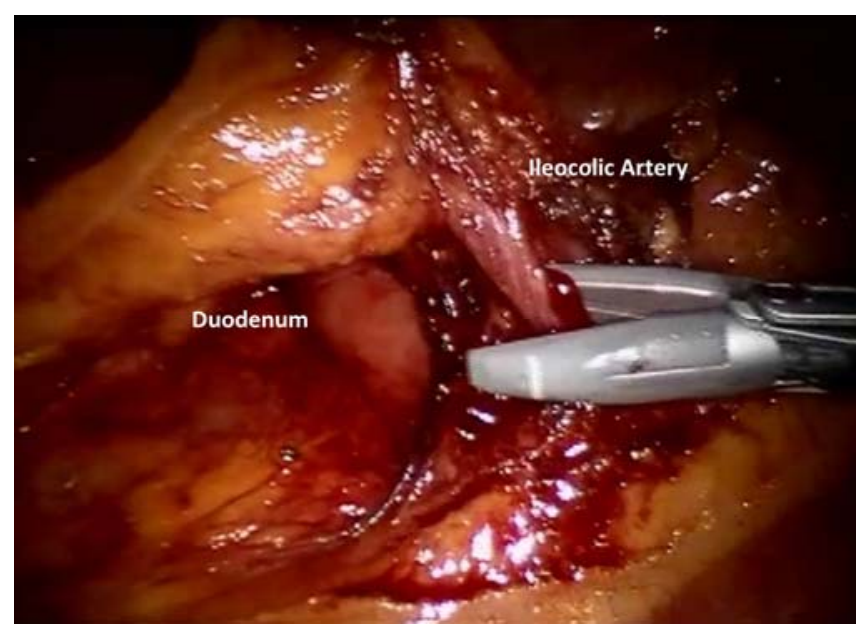

Figure 8

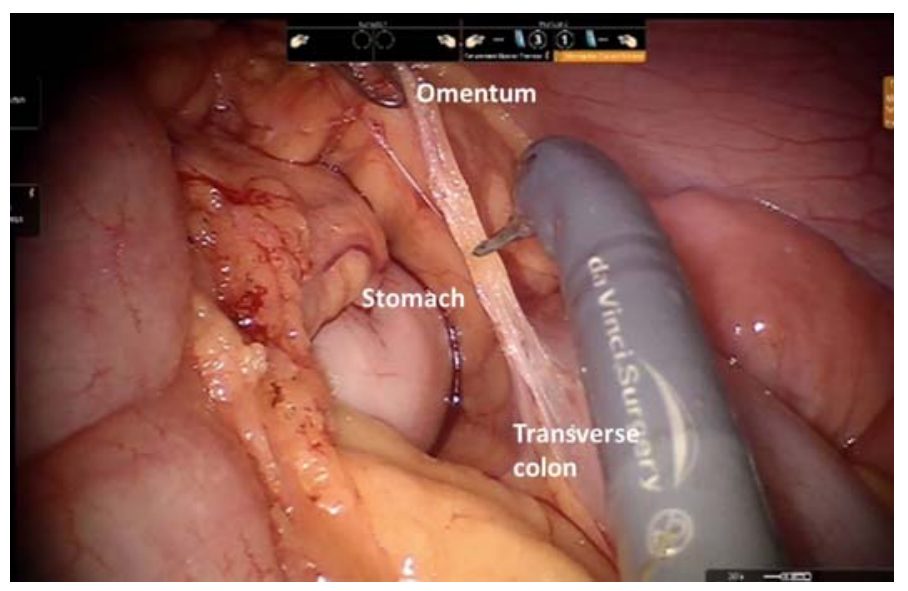

Figure 9 


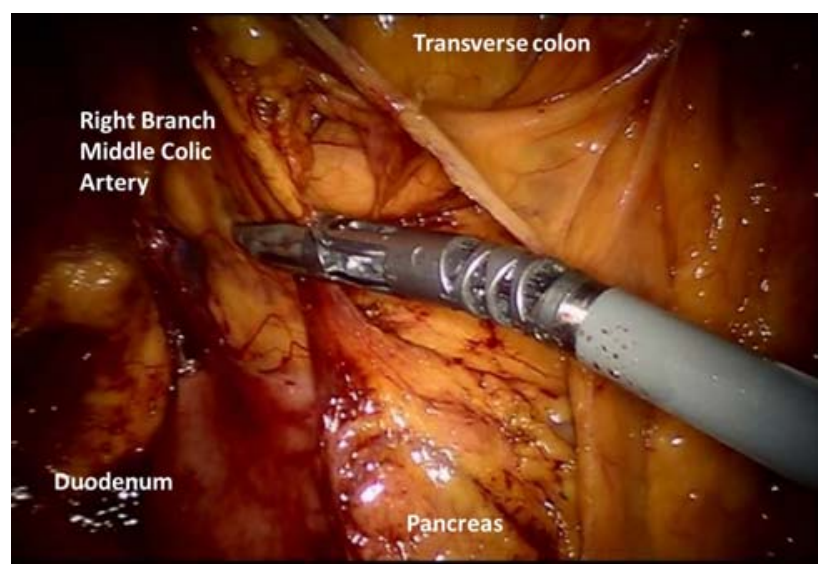

Figure 10

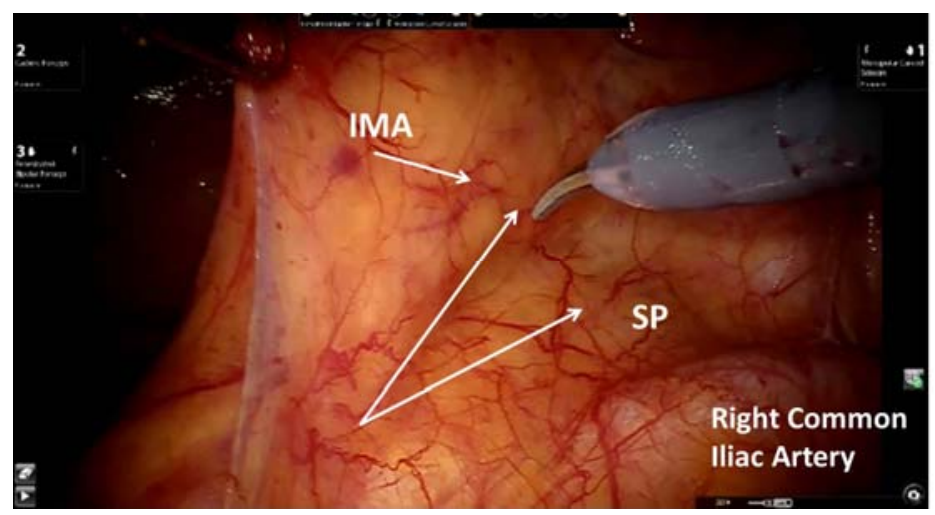

Figure 11

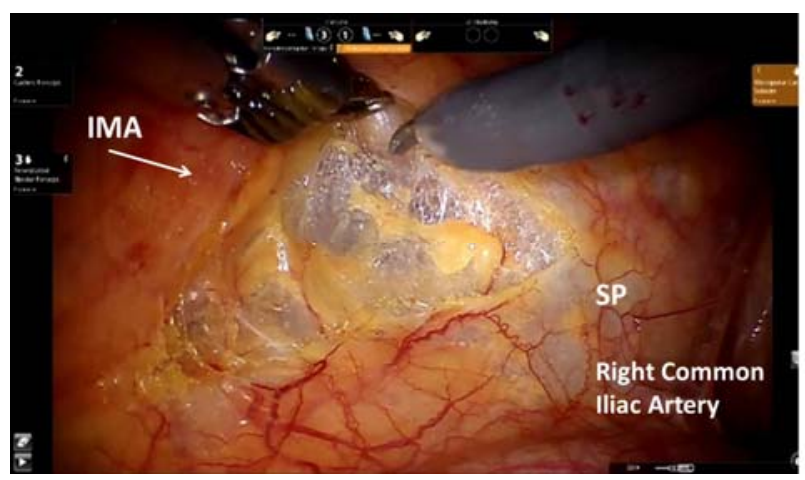

Figure 12 


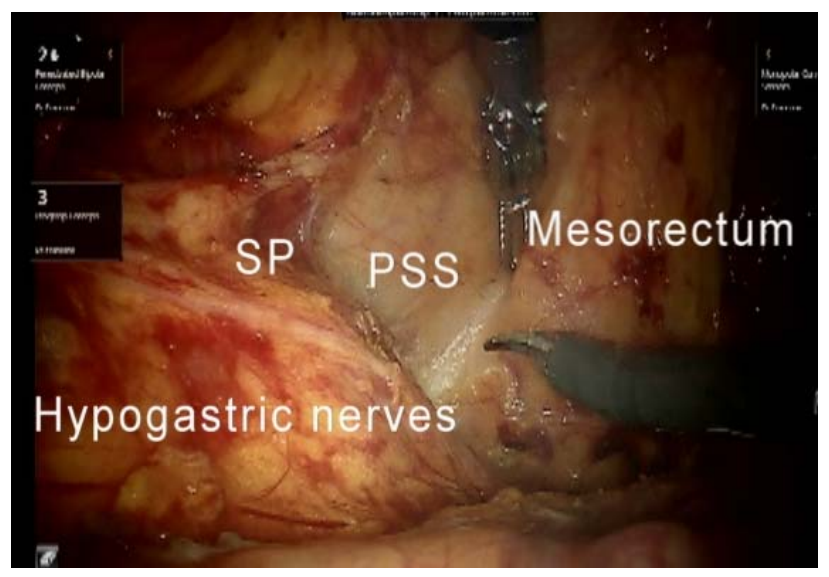

Figure 13

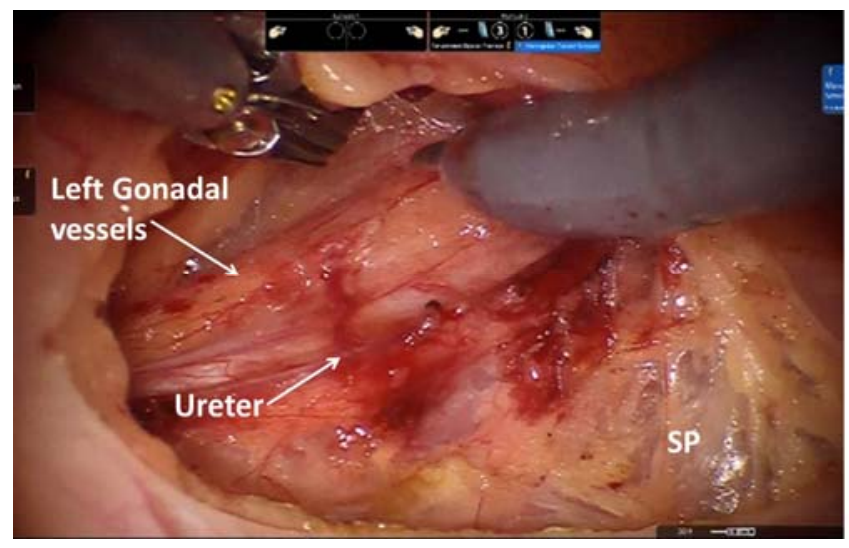

Figure 14

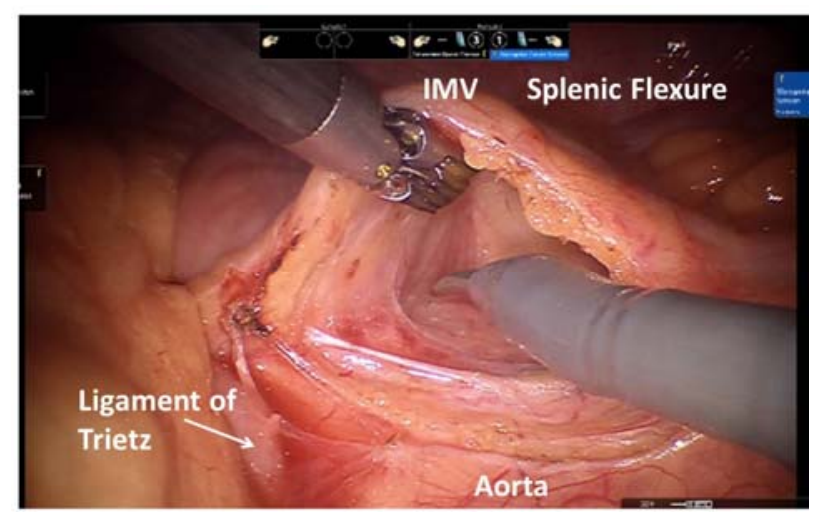

Figure 15 


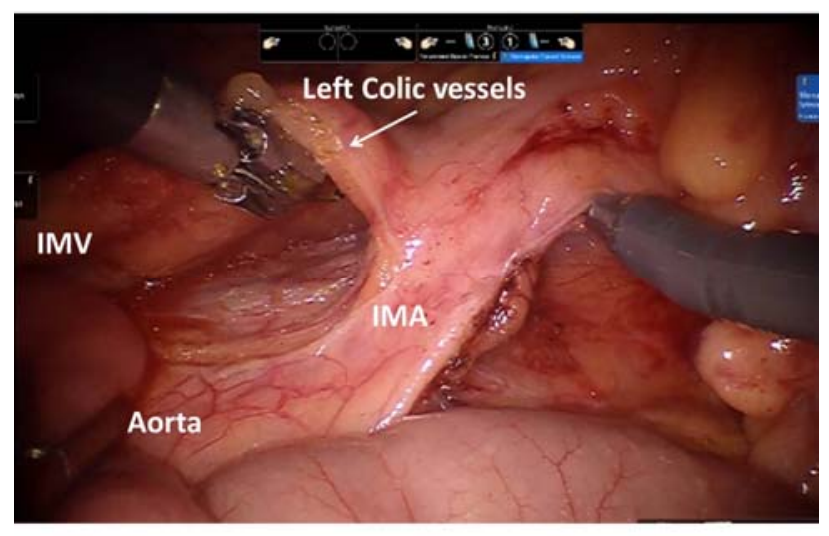

Figure 16

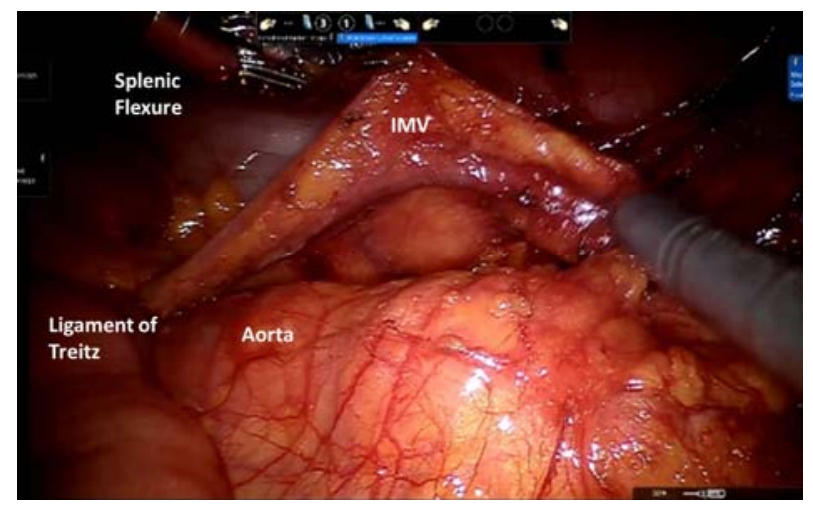

Figure 17

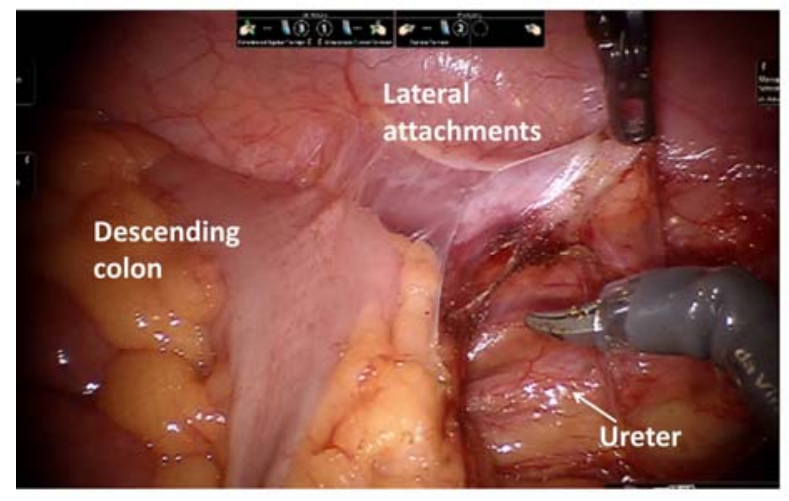

Figure 18 